\title{
Size-dependent Correlation Effects in Ultrafast Optical Dynamics of Metal Nanoparticles
}

\author{
T. V. Shahbazyan and I. E. Perakis \\ Department of Physics and Astronomy, Vanderbilt University, Box 1807-B, Nashville, TN 37235
}

\begin{abstract}
We study the role of collective surface excitations in the electron relaxation in small metal particles. We show that the dynamically screened electronelectron interaction in a nanoparticle contains a size-dependent correction induced by the surface. This leads to new channels of quasiparticle scattering accompanied by the emission of surface collective excitations. We calculate the energy and temperature dependence of the corresponding rates, which depend strongly on the nanoparticle size. We show that the surface-plasmonmediated scattering rate of a conduction electron increases with energy, in contrast to that mediated by a bulk plasmon. In noble-metal particles, we find that the dipole collective excitations (surface plasmons) mediate a resonant scattering of $d$-holes to the conduction band. We study the role of the latter effect in the ultrafast optical dynamics of small nanoparticles and show that, with decreasing nanoparticle size, it leads to a drastic change in the differential absorption lineshape and a strong frequency dependence of the relaxation near the surface plasmon resonance. The experimental implications of our results in ultrafast pump-probe spectroscopy are also discussed.

Pacs numbers: 36.40.Gk, 36.40.Vz, 61.46.+w, 78.47.+p
\end{abstract}

Typeset using REVTEX 


\section{INTRODUCTION}

The properties of small metal particles in the intermediate regime between bulk-like and molecular behavior have been a subject of great interest recently. 1 - 1 Even though the electronic and optical properties of nanoparticles have been extensively studied, the effect of confinement on electron dynamics is much less understood. Examples of outstanding issues include the role of electron-electron interactions in the process of cluster fragmentation, the role of surface lattice modes in providing additional channels for intra-molecular energy relaxation, the influence of the electron and nuclear motion on the superparamagnetic properties of clusters, and the effect of confinement on the nonlinear optical properties and transient response under ultrafast excitation. be studied with femtosecond nonlinear optical spectroscopy, which allows one to probe the time evolution of the excited states with a resolution shorter than the energy relaxation or dephasing times.

Surface collective excitations play an important role in the absorption of light by metal nanoparticles. In large particles with sizes comparable to the wave-length of light $\lambda$ (but smaller than the bulk mean free path), the lineshape of the surface plasmon (SP) resonance is determined by the electromagnetic effects 1 In small nanoparticles with radii $R \ll \lambda$, the absorption spectrum is governed by quantum confinement effects. For example, the momentum non-conservation due to the confining potential leads to the Landau damping of the SP and to a resonance linewidth inversely proportional to the nanoparticle size. $\mathrm{\theta}$.: Confinement changes also non-linear optical properties of nanoparticles: a size-dependent enhancement of the third order susceptibilities, caused by the elastic surface scattering of single-particle excitations, has been reported.6

Extensive experimental studies of the electron relaxation in nanoparticles have recently been performed using ultrafast pump-probe spectroscopy 9 - 6 Unlike in semiconductors, the dephasing processes in metals are very fast, and nonequilibrium populations of optically excited electrons and holes are formed within several femtoseconds. These thermalize into 
the hot Fermi-Dirac distribution within several hundreds of femtoseconds, mainly due to $e-e$ and $h-h$ scattering.1720 Since the electron heat capacity is much smaller than that of the lattice, a high electron temperature can be reached during less than 1 ps time scales, i.e., before any significant energy transfer to the phonon bath occurs. During this stage, the SP resonance was observed to undergo a time-dependent spectral broadening. 11.13 Subsequently, the electron and phonon baths equilibrate through the electron-phonon interactions over time intervals of a few picoseconds. During this incoherent stage, the hot electron distribution can be characterized by a time-dependent temperature. Correlation effects play an important role in the latter regime. For example, in order to explain the differential absorption lineshape, it is essential to take into account the $e-e$ scattering of the optically-excited carriers near the Fermi surface.11 Furthermore, despite the similarities to the bulk-like behavior, observed, e.g., in metal films, certain aspects of the optical dynamics in nanoparticles are significantly different.14.11 16 For example, experimental studies of small Cu nanoparticles revealed that the relaxation times of the the pump-probe signal depend strongly on frequency: the relaxation was considerably slower at the SP resonance.11, 16 This and other observations suggest that collective surface excitations play an important role in the electron dynamics in small metal particles.

Let us recall the basic facts regarding the linear absorption by metal nanoparticles embedded in a medium with dielectric constant $\epsilon_{m}$. We will focus primarily on noble metal particles containing several hundreds of atoms; in this case, the confinement affects the extended electronic states even though the bulk lattice structure has been established. When the particles radii are small, $R \ll \lambda$, so that only dipole surface modes can be optically excited and non-local effects can be neglected, the optical properties of this system are determined by the dielectric function 1

$$
\epsilon_{\mathrm{col}}(\omega)=\epsilon_{m}+3 p \epsilon_{m} \frac{\epsilon(\omega)-\epsilon_{m}}{\epsilon(\omega)+2 \epsilon_{m}}
$$

where $\epsilon(\omega)=\epsilon^{\prime}(\omega)+i \epsilon^{\prime \prime}(\omega)$ is the dielectric function of a metal particle and $p \ll 1$ is the volume fraction occupied by nanoparticles in the colloid. Since the $d$-electrons play an 
important role in the optical properties of noble metals, the dielectric function $\epsilon(\omega)$ includes also the interband contribution $\epsilon_{d}(\omega)$. For $p \ll 1$, the absorption coefficient of such a system is proportional to that of a single particle and is given by

$$
\alpha(\omega)=-9 p \epsilon_{m}^{3 / 2} \frac{\omega}{c} \operatorname{Im} \frac{1}{\epsilon_{s}(\omega)}
$$

where

$$
\epsilon_{s}(\omega)=\epsilon_{d}(\omega)-\omega_{p}^{2} / \omega\left(\omega+i \gamma_{s}\right)+2 \epsilon_{m}
$$

plays the role of an effective dielectric function of a particle in the medium. Its zero, $\epsilon_{s}^{\prime}\left(\omega_{s}\right)=0$, determines the frequency of the SP, $\omega_{s}$. In Eq. (3), $\omega_{p}$ is the bulk plasmon frequency of the conduction electrons, and the width $\gamma_{s}$ characterizes the SP damping. The semiclassical result Eqs. (2) and (3) applies to nanoparticles with radii $R \gg q_{T F}^{-1}$, where $q_{T F}$ is the Thomas-Fermi screening wave-vector $\left(q_{T F}^{-1} \sim 1 \AA\right.$ in noble metals). In this case, the electron density deviates from its classical shape only within a surface layer occupying a small fraction of the total volume.21 Quantum mechanical corrections, arising from the discrete energy spectrum, lead to a width $\gamma_{s} \sim v_{F} / R$, where $v_{F}=k_{F} / m$ is the Fermi velocity. . Even though $\gamma_{s} / \omega_{s} \sim\left(q_{T F} R\right)^{-1} \ll 1$, this damping mechanism dominates over others, e.g., due to phonons, for sizes $R \lesssim 10 \mathrm{~nm}$. In small clusters, containing several dozens of atoms, the semiclassical approximation breaks down and density functional or ab initio methods should be used.t.

It should be noted that, in contrast to surface collective excitations, the $e-e$ scattering is not sensitive to the nanoparticle size as long as the condition $q_{T F} R \gg 1$ holds.22 Indeed, for such sizes, the static screening is essentially bulk-like. At the same time, the energy dependence of the bulk $e-e$ scattering rate, ${ }^{23} \gamma_{e} \propto\left(E-E_{F}\right)^{2}$, with $E_{F}$ being the Fermi energy, comes from the phase-space restriction due to the momentum conservation, and involves the exchange of typical momenta $q \sim q_{T F}$. If the size-induced momentum uncertainty $\delta q \sim R^{-1}$ is much smaller than $q_{T F}$, the $e-e$ scattering rate in a nanoparticle is not significantly affected by the confinement 24 
In this paper we address the role of collective surface excitations in the electron relaxation in small metal particles. We show that the dynamically screened $e-e$ interaction contains a correction originating from the surface collective modes excited by an electron in nanoparticle. This opens up new quasiparticle scattering channels mediated by surface collective modes. We derive the corresponding scattering rates, which depend strongly on the nanoparticle size. The scattering rate of a conduction electron increases with energy, in contrast to the bulk-plasmon mediated scattering. In noble metal particles, we study the $\mathrm{SP}$-mediated scattering of a $d$-hole into the conduction band. The scattering rate of this process depends strongly on temperature, and exhibits a peak as a function of energy due to the restricted phase space available for interband scattering. We show that this effect manifests itself in the ultrafast nonlinear optical dynamics of nanometer-sized particles. In particular, our self-consistent calculations show that, near the SP resonance, the differential absorption lineshape undergoes a dramatic transformation as the particle size decreases. We also find that the relaxation times of the pump-probe signal depend strongly on the probe frequency, in agreement with recent experiments.

The paper is organized as follows. In Section $\mathbb{\text { II }}$ we derive the dynamically screened Coulomb potential in a nanoparticle. In Section $[1]$ we calculate the SP-mediated quasiparticle scattering rates of the conduction electrons and the $d$-band holes. In Section IV we incorporate these effects in the calculation of the absorption spectrum and study their role in the size and frequency dependence of the time-resolved pump-probe signal.

\section{ELECTRON-ELECTRON INTERACTIONS IN METAL NANOPARTICLES}

In this section, we study the effect of the surface collective excitations on the $e-e$ inter-

actions in a spherical metal particle. To find the dynamically screened Coulomb potential, we generalize the method previously developed for calculations of local field corrections to the optical fields.25 The potential $U\left(\omega ; \mathbf{r}, \mathbf{r}^{\prime}\right)$ at point $\mathbf{r}$ arising from an electron at point $\mathbf{r}^{\prime}$ is determined by the equation 26 


$$
U\left(\omega ; \mathbf{r}, \mathbf{r}^{\prime}\right)=u\left(\mathbf{r}-\mathbf{r}^{\prime}\right)+\int d \mathbf{r}_{1} d \mathbf{r}_{2} u\left(\mathbf{r}-\mathbf{r}_{1}\right) \Pi\left(\omega ; \mathbf{r}_{1}, \mathbf{r}_{2}\right) U\left(\omega ; \mathbf{r}_{2}, \mathbf{r}^{\prime}\right)
$$

where $u\left(\mathbf{r}-\mathbf{r}^{\prime}\right)=e^{2}\left|\mathbf{r}-\mathbf{r}^{\prime}\right|^{-1}$ is the unscreened Coulomb potential and $\Pi\left(\omega ; \mathbf{r}_{1}, \mathbf{r}_{2}\right)$ is the polarization operator. There are three contributions to $\Pi$, arising from the polarization of the conduction electrons, the $d$-electrons, and the medium surrounding the nanoparticles: $\Pi=\Pi_{c}+\Pi_{d}+\Pi_{m}$. It is useful to rewrite Eq. (田) in the "classical" form

$$
\nabla \cdot(\mathbf{E}+4 \pi \mathbf{P})=4 \pi e^{2} \delta\left(\mathbf{r}-\mathbf{r}^{\prime}\right)
$$

where $\mathbf{E}\left(\omega ; \mathbf{r}, \mathbf{r}^{\prime}\right)=-\nabla U\left(\omega ; \mathbf{r}, \mathbf{r}^{\prime}\right)$ is the screened Coulomb field and $\mathbf{P}=\mathbf{P}_{c}+\mathbf{P}_{d}+\mathbf{P}_{m}$ is the electric polarization vector, related to the potential $U$ as

$$
\nabla \mathbf{P}\left(\omega ; \mathbf{r}, \mathbf{r}^{\prime}\right)=-e^{2} \int d \mathbf{r}_{1} \Pi\left(\omega ; \mathbf{r}, \mathbf{r}_{1}\right) U\left(\omega ; \mathbf{r}_{1}, \mathbf{r}^{\prime}\right)
$$

In the random phase approximation, the intraband polarization operator is given by

$$
\Pi_{c}\left(\omega ; \mathbf{r}, \mathbf{r}^{\prime}\right)=\sum_{\alpha \alpha^{\prime}} \frac{f\left(E_{\alpha}^{c}\right)-f\left(E_{\alpha^{\prime}}^{c}\right)}{E_{\alpha}^{c}-E_{\alpha^{\prime}}^{c}+\omega+i 0} \psi_{\alpha}^{c}(\mathbf{r}) \psi_{\alpha^{\prime}}^{c *}(\mathbf{r}) \psi_{\alpha}^{c *}\left(\mathbf{r}^{\prime}\right) \psi_{\alpha^{\prime}}^{c}\left(\mathbf{r}^{\prime}\right),
$$

where $E_{\alpha}^{c}$ and $\psi_{\alpha}^{c}$ are the single-electron eigenenergies and eigenfunctions in the nanoparticle, and $f(E)$ is the Fermi-Dirac distribution (we set $\hbar=1$ ). Since we are interested in frequencies much larger than the single-particle level spacing, $\Pi_{c}(\omega)$ can be expanded in terms of $1 / \omega$. For the real part, $\Pi_{c}^{\prime}(\omega)$, we obtain in the leading order 5

$$
\Pi_{c}^{\prime}\left(\omega ; \mathbf{r}, \mathbf{r}_{1}\right)=-\frac{1}{m \omega^{2}} \nabla\left[n_{c}(\mathbf{r}) \nabla \delta\left(\mathbf{r}-\mathbf{r}_{1}\right)\right]
$$

where $n_{c}(\mathbf{r})$ is the conduction electron density. In the following we assume, for simplicity, a step density profile, $n_{c}(\mathbf{r})=\bar{n}_{c} \theta(R-r)$, where $\bar{n}_{c}$ is the average density. The leading contribution to the imaginary part, $\Pi_{c}^{\prime \prime}(\omega)$, is proportional to $\omega^{-3}$, so that $\Pi_{c}^{\prime \prime}(\omega) \ll \Pi_{c}^{\prime}(\omega)$.

By using Eqs. (8) and (6), one obtains a familiar expression for $\mathbf{P}_{c}$ at high frequencies,

$$
\mathbf{P}_{c}\left(\omega ; \mathbf{r}, \mathbf{r}^{\prime}\right)=\frac{e^{2} n_{c}(\mathbf{r})}{m \omega^{2}} \nabla U\left(\omega ; \mathbf{r}, \mathbf{r}^{\prime}\right)=\theta(R-r) \chi_{c}(\omega) \mathbf{E}\left(\omega ; \mathbf{r}, \mathbf{r}^{\prime}\right)
$$

where $\chi_{c}(\omega)=-e^{2} \bar{n}_{c} / m \omega^{2}$ is the conduction electron susceptibility. Note that, for a step density profile, $\mathbf{P}_{c}$ vanishes outside the particle. The $d$-band and dielectric medium contributions to $\mathbf{P}$ are also given by similar relations, 


$$
\begin{gathered}
\mathbf{P}_{d}\left(\omega ; \mathbf{r}, \mathbf{r}^{\prime}\right)=\theta(R-r) \chi_{d}(\omega) \mathbf{E}\left(\omega ; \mathbf{r}, \mathbf{r}^{\prime}\right) \\
\mathbf{P}_{m}\left(\omega ; \mathbf{r}, \mathbf{r}^{\prime}\right)=\theta(r-R) \chi_{m} \mathbf{E}\left(\omega ; \mathbf{r}, \mathbf{r}^{\prime}\right)
\end{gathered}
$$

where $\chi_{i}=\left(\epsilon_{i}-1\right) / 4 \pi, i=d, m$ are the corresponding susceptibilities and the step functions account for the boundary conditions.27 Using Eqs. (9)-(11), one can write a closed equation for $U\left(\omega ; \mathbf{r}, \mathbf{r}^{\prime}\right)$. Using Eq. (6), the second term of Eq. (4) can be presented as $-e^{-2} \int d \mathbf{r}_{1} u(\mathbf{r}-$ $\left.\mathbf{r}_{1}\right) \nabla \cdot \mathbf{P}\left(\omega ; \mathbf{r}_{1}, \mathbf{r}^{\prime}\right)$. Substituting the above expressions for $\mathbf{P}$, we then obtain after integrating by parts

$$
\begin{gathered}
\epsilon(\omega) U\left(\omega ; \mathbf{r}, \mathbf{r}^{\prime}\right)=\frac{e^{2}}{\left|\mathbf{r}-\mathbf{r}^{\prime}\right|}+\int d \mathbf{r}_{1} \nabla_{1} \frac{1}{\left|\mathbf{r}-\mathbf{r}_{1}\right|} \cdot \nabla_{1}\left[\theta(R-r) \chi(\omega)+\theta(r-R) \chi_{m}\right] U\left(\omega ; \mathbf{r}_{1}, \mathbf{r}^{\prime}\right) \\
+i \int d \mathbf{r}_{1} d \mathbf{r}_{2} \frac{e^{2}}{\left|\mathbf{r}-\mathbf{r}_{1}\right|} \Pi_{c}^{\prime \prime}\left(\omega ; \mathbf{r}_{1}, \mathbf{r}_{2}\right) U\left(\omega ; \mathbf{r}_{2}, \mathbf{r}^{\prime}\right)
\end{gathered}
$$

with

$$
\epsilon(\omega) \equiv 1+4 \pi \chi(\omega)=\epsilon_{d}(\omega)-\omega_{p}^{2} / \omega^{2}
$$

$\omega_{p}^{2}=4 \pi e^{2} \bar{n}_{c} / m$ being the plasmon frequency in the conduction band. The last term in

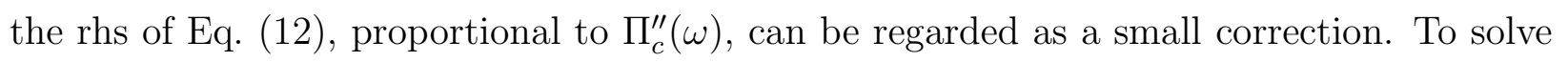
Eq. (12), we first eliminate the angular dependence by expanding $U\left(\omega ; \mathbf{r}, \mathbf{r}^{\prime}\right)$ in spherical harmonics, $Y_{L M}(\hat{\mathbf{r}})$, with coefficients $U_{L M}\left(\omega ; r, r^{\prime}\right)$. Using the corresponding expansion of $\left|\mathbf{r}-\mathbf{r}^{\prime}\right|^{-1}$ with coefficients $Q_{L M}\left(r, r^{\prime}\right)=\frac{4 \pi}{2 L+1} r^{-L-1} r^{\prime L}$ (for $r>r^{\prime}$ ), we get the following equation for $U_{L M}\left(\omega ; r, r^{\prime}\right)$ :

$$
\begin{aligned}
\epsilon(\omega) U_{L M}\left(\omega ; r, r^{\prime}\right)= & Q_{L M}\left(r, r^{\prime}\right)+4 \pi\left[\chi(\omega)-\chi_{m}\right] \frac{L+1}{2 L+1}\left(\frac{r}{R}\right)^{L} U_{L M}\left(\omega ; R, r^{\prime}\right) \\
& +i e^{2} \sum_{L^{\prime} M^{\prime}} \int d r_{1} d r_{2} r_{1}^{2} r_{2}^{2} Q_{L M}\left(r, r_{1}\right) \Pi_{L M, L^{\prime} M^{\prime}}^{\prime \prime}\left(\omega ; r_{1}, r_{2}\right) U_{L^{\prime} M^{\prime}}\left(\omega ; r_{2}, r^{\prime}\right)
\end{aligned}
$$

where

$$
\Pi_{L M, L^{\prime} M^{\prime}}^{\prime \prime}\left(\omega ; r_{1}, r_{2}\right)=\int d \hat{\mathbf{r}}_{1} d \hat{\mathbf{r}}_{2} Y_{L M}^{*}\left(\hat{\mathbf{r}}_{1}\right) \Pi_{c}^{\prime \prime}\left(\omega ; \mathbf{r}_{1}, \mathbf{r}_{2}\right) Y_{L^{\prime} M^{\prime}}\left(\hat{\mathbf{r}}_{2}\right)
$$

are the coefficients of the multipole expansion of $\Pi_{c}^{\prime \prime}\left(\omega ; \mathbf{r}_{1}, \mathbf{r}_{2}\right)$. For $\Pi_{c}^{\prime \prime}=0$, the solution of Eq. (14) can be presented in the form 


$$
U_{L M}\left(\omega ; r, r^{\prime}\right)=a(\omega) e^{2} Q_{L M}\left(r, r^{\prime}\right)+b(\omega) \frac{4 \pi e^{2}}{2 L+1} \frac{r^{L} r^{\prime L}}{R^{2 L+1}}
$$

with frequency-dependent coefficients $a$ and $b$. Since $\Pi_{c}^{\prime \prime}(\omega) \ll \Pi_{c}^{\prime}(\omega)$ for relevant frequencies, the solution of Eq. (14) in the presence of the last term can be written in the same form as Eq. (16), but with modified $a(\omega)$ and $b(\omega)$. Substituting Eq. (16) into Eq. (14), we obtain after lengthy algebra in the lowest order in $\Pi_{c}^{\prime \prime}$

$$
a(\omega)=\epsilon^{-1}(\omega), \quad b(\omega)=\epsilon_{L}^{-1}(\omega)-\epsilon^{-1}(\omega)
$$

where

$$
\epsilon_{L}(\omega)=\frac{L}{2 L+1} \epsilon(\omega)+\frac{L+1}{2 L+1} \epsilon_{m}+i \epsilon_{c L}^{\prime \prime}(\omega)
$$

is the effective dielectric function, whose zero, $\epsilon_{L}^{\prime}\left(\omega_{L}\right)=0$, determines the frequency of the collective surface excitation with angular momentum $L$,

$$
\omega_{L}^{2}=\frac{L \omega_{p}^{2}}{L \epsilon_{d}^{\prime}\left(\omega_{L}\right)+(L+1) \epsilon_{m}} .
$$

In Eq. (18), $\epsilon_{c L}^{\prime \prime}(\omega)$ characterizes the damping of the $L$-pole collective mode by single-particle excitations, and is given by

$$
\epsilon_{c L}^{\prime \prime}(\omega)=\frac{4 \pi^{2} e^{2}}{(2 L+1) R^{2 L+1}} \sum_{\alpha \alpha^{\prime}}\left|M_{\alpha \alpha^{\prime}}^{L M}\right|^{2}\left[f\left(E_{\alpha}^{c}\right)-f\left(E_{\alpha^{\prime}}^{c}\right)\right] \delta\left(E_{\alpha}^{c}-E_{\alpha^{\prime}}^{c}+\omega\right),
$$

where $M_{\alpha \alpha^{\prime}}^{L M}$ are the matrix elements of $r^{L} Y_{L M}(\hat{\mathbf{r}})$. Due to the momentum nonconservation in a nanoparticle, the matrix elements are finite, which leads to the size-dependent width of the $L$-pole mode:5 25

$$
\gamma_{L}=\frac{2 L+1}{L} \frac{\omega^{3}}{\omega_{p}^{2}} \epsilon_{c L}^{\prime \prime}(\omega)
$$

For $\omega \sim \omega_{L}$, one can show that the width, $\gamma_{L} \sim v_{F} / R$, is independent of $\omega$. Note that, in noble metal particles, there is an additional $d$-electron contribution to the imaginary part of $\epsilon_{L}(\omega)$ at frequencies above the onset $\Delta$ of the interband transitions.

Putting everything together, we arrive at the following expression for the dynamicallyscreened interaction potential in a nanoparticle: 


$$
U\left(\omega ; \mathbf{r}, \mathbf{r}^{\prime}\right)=\frac{u\left(\mathbf{r}-\mathbf{r}^{\prime}\right)}{\epsilon(\omega)}+\frac{e^{2}}{R} \sum_{L M} \frac{4 \pi}{2 L+1} \frac{1}{\tilde{\epsilon}_{L}(\omega)}\left(\frac{r r^{\prime}}{R^{2}}\right)^{L} Y_{L M}(\hat{\mathbf{r}}) Y_{L M}^{*}\left(\hat{\mathbf{r}}^{\prime}\right),
$$

with $\tilde{\epsilon}_{L}^{-1}(\omega)=\epsilon_{L}^{-1}(\omega)-\epsilon^{-1}(\omega)$. Equation (22), which is the main result of this section, represents a generalization of the plasmon pole approximation to spherical particles. The two terms in the rhs describe two distinct contributions. The first comes from the usual bulk-like screening of the Coulomb potential. The second contribution describes a new effective $e-e$ interaction induced by the surface: the potential of an electron inside the nanoparticle excites high-frequency surface collective modes, which in turn act as image charges that interact with the second electron. It should be emphasized that, unlike in the case of the optical fields, the surface-induced dynamical screening of the Coulomb potential is size-dependent.

Note that the excitation energies of the surface collective modes are lower than the bulk plasmon energy, also given by Eq. (19) but with $\epsilon_{m}=0$. This opens up new channels of quasiparticle scattering, considered in the next section.

\section{QUASIPARTICLE SCATTERING VIA SURFACE COLLECTIVE MODES}

In this section we calculate the rates of quasiparticle scattering accompanied by the emission of surface collective modes. We start with the scattering of an electron in the conduction band. In the first order in the surface-induced potential, given by the second term in the rhs of Eq. (22), the corresponding scattering rate can be obtained from the Matsubara self-energy 2

$$
\Sigma_{\alpha}^{c}(i \omega)=-\frac{1}{\beta} \sum_{i \omega^{\prime}} \sum_{L M} \sum_{\alpha^{\prime}} \frac{4 \pi e^{2}}{(2 L+1) R^{2 L+1}} \frac{\left|M_{\alpha \alpha^{\prime}}^{L M}\right|^{2}}{\tilde{\epsilon}_{L}\left(i \omega^{\prime}\right)} G_{\alpha^{\prime}}^{c}\left(i \omega^{\prime}+i \omega\right),
$$

where $G_{\alpha}^{c}=\left(i \omega-E_{\alpha}^{c}\right)^{-1}$ is the non-interacting Green function of the conduction electron. Here the matrix elements $M_{\alpha \alpha^{\prime}}^{L M}$ are calculated with the one-electron wave functions $\psi_{\alpha}^{c}(\mathbf{r})=$ $R_{n l}(r) Y_{l m}(\hat{\mathbf{r}})$. Since $|\alpha\rangle$ and $\left|\alpha^{\prime}\right\rangle$ are the initial and final states of the scattered electron, the main contribution to the $L$ th term of the angular momentum sum in Eq. (23) will come from

electron states with energy difference $E_{\alpha}-E_{\alpha^{\prime}} \sim \omega_{L}$. Therefore, $M_{\alpha \alpha^{\prime}}^{L M}$ can be expanded 
in terms of the small parameter $E_{0} /\left|E_{\alpha}^{c}-E_{\alpha^{\prime}}^{c}\right| \sim E_{0} / \omega_{L}$, where $E_{0}=\left(2 m R^{2}\right)^{-1}$ is the characteristic confinement energy. The leading term can be obtained by using the following procedure. 2.25 We present $M_{\alpha \alpha^{\prime}}^{L M}$ as

$$
M_{\alpha \alpha^{\prime}}^{L M}=\left\langle c, \alpha\left|r^{L} Y_{L M}(\hat{\mathbf{r}})\right| c, \alpha^{\prime}\right\rangle=\frac{\left\langle c, \alpha\left|\left[H,\left[H, r^{L} Y_{L M}(\hat{\mathbf{r}})\right]\right]\right| c, \alpha^{\prime}\right\rangle}{\left(E_{\alpha}^{c}-E_{\alpha^{\prime}}^{c}\right)^{2}},
$$

where $H=H_{0}+V(r)$ is the Hamiltonian of an electron in a nanoparticle with confining potential $V(r)=V_{0} \theta(r-R)$. Since $\left[H, r^{L} Y_{L M}(\hat{\mathbf{r}})\right]=-\frac{1}{m} \nabla\left[r^{L} Y_{L M}(\hat{\mathbf{r}})\right] \cdot \nabla$, the numerator in Eq. (24) contains a term proportional to the gradient of the confining potential, which peaks sharply at the surface. The corresponding contribution to the matrix element describes the surface scattering of an electron making the $L$-pole transition between the states $|c, \alpha\rangle$ and $\left|c, \alpha^{\prime}\right\rangle$, and gives the dominant term of the expansion. Thus, in the leading order in $\left|E_{\alpha}^{c}-E_{\alpha^{\prime}}^{c}\right|^{-1}$, we obtain

$$
M_{\alpha \alpha^{\prime}}^{L M}=\frac{\left\langle c, \alpha\left|\nabla\left[r^{L} Y_{L M}(\hat{\mathbf{r}})\right] \cdot \nabla V(r)\right| c, \alpha^{\prime}\right\rangle}{m\left(E_{\alpha}^{c}-E_{\alpha^{\prime}}^{c}\right)^{2}}=\frac{L R^{L+1}}{m\left(E_{\alpha}^{c}-E_{\alpha^{\prime}}^{c}\right)^{2}} V_{0} R_{n l}(R) R_{n^{\prime} l^{\prime}}(R) \varphi_{l m, l^{\prime} m^{\prime}}^{L M},
$$

with $\varphi_{l m, l^{\prime} m^{\prime}}^{L M}=\int d \hat{\mathbf{r}} Y_{l m}^{*}(\hat{\mathbf{r}}) Y_{L M}(\hat{\mathbf{r}}) Y_{l^{\prime} m^{\prime}}(\hat{\mathbf{r}})$. Note that, for $L=1$, Eq. (25) becomes exact. For electron energies close to the Fermi level, $E_{n l}^{c} \sim E_{F}$, the radial quantum numbers are large, and the product $V_{0} R_{n l}(R) R_{n^{\prime} l^{\prime}}(R)$ can be evaluated by using semiclassical wave-functions. In the limit $V_{0} \rightarrow \infty$, this product is given by $2 \sqrt{E_{n l}^{c} E_{n^{\prime} l^{\prime}}^{c}} / R^{3}$, where $E_{n l}^{c}=\pi^{2}(n+l / 2)^{2} E_{0}$ is the electron eigenenergy for large $n$. Substituting this expression into Eq. (25) and then into Eq. (23), we obtain

$$
\Sigma_{\alpha}^{c}(i \omega)=-\frac{1}{\beta} \sum_{i \omega^{\prime}} \sum_{L} \sum_{n^{\prime} l^{\prime}} C_{l l^{\prime}}^{L} \frac{4 \pi e^{2}}{(2 L+1) R} \frac{E_{n l}^{c} E_{n^{\prime} l^{\prime}}^{c}}{\left(E_{n l}^{c}-E_{n^{\prime} l^{\prime}}^{c}\right)^{4}} \frac{\left(4 L E_{0}\right)^{2}}{\tilde{\epsilon}_{L}\left(i \omega^{\prime}\right)} G_{\alpha^{\prime}}^{c}\left(i \omega^{\prime}+i \omega\right),
$$

with

$$
C_{l l^{\prime}}^{L}=\sum_{M, m^{\prime}}\left|\varphi_{l m, l^{\prime} m^{\prime}}^{L M}\right|^{2}=\frac{(2 L+1)\left(2 l^{\prime}+1\right)}{8 \pi} \int_{-1}^{1} d x P_{l}(x) P_{L}(x) P_{l^{\prime}}(x)
$$

where $P_{l}(x)$ are Legendre polynomials; we used properties of the spherical harmonics in the derivation of Eq. (27). For $E_{n l}^{c} \sim E_{F}$, the typical angular momenta are large, $l \sim k_{F} R \gg 1$, and one can use the large $-l$ asymptotics of $P_{l}$; for the low multipoles of interest, $L \ll l$, 
the integral in Eq. (27) can be approximated by $\frac{2}{2 l^{\prime}+1} \delta_{l l^{\prime}}$. After performing the Matsubara summation, we obtain for the imaginary part of the self-energy that determines the electron scattering rate

$$
\operatorname{Im} \Sigma_{\alpha}^{c}(\omega)=-\frac{16 e^{2}}{R} E_{0}^{2} \sum_{L} L^{2} \int d E g_{l}(E) \frac{E E_{\alpha}^{c}}{\left(E_{\alpha}^{c}-E\right)^{4}} \operatorname{Im} \frac{N(E-\omega)+f(E)}{\tilde{\epsilon}_{L}(E-\omega)},
$$

where $N(E)$ is the Bose distribution and $g_{l}(E)$ is the density of states of a conduction electron with angular momentum $l$,

$$
g_{l}(E)=2 \sum_{n} \delta\left(E_{n l}^{c}-E\right) \simeq \frac{R}{\pi} \sqrt{\frac{2 m}{E}}
$$

where we replaced the sum over $n$ by an integral (the factor of 2 accounts for spin).

Each term in the sum in the rhs of Eq. (28) represents a channel of electron scattering mediated by a collective surface mode with angular momentum $L$. For low $L$, the difference between the energies of modes with successive values of $L$ is larger than their widths, so that the different channels are well separated. Note that since all $\omega_{L}$ are smaller than the frequency of the (undamped) bulk plasmon, one can replace $\tilde{\epsilon}_{L}(\omega)$ by $\epsilon_{L}(\omega)$ in the integrand of Eq. (28) for frequencies $\omega \sim \omega_{L}$.

Consider now the $L=1$ term in Eq. (28), which describes the SP-mediated scattering channel. The main contribution to the integral comes from the SP pole in $\epsilon_{1}^{-1}(\omega)=3 \epsilon_{s}^{-1}(\omega)$, where $\epsilon_{s}(\omega)$ is the same as in Eq. (3). To estimate the scattering rate, we approximate $\operatorname{Im} \epsilon_{s}^{-1}(\omega)$ by a Lorentzian,

$$
\operatorname{Im} \epsilon_{s}^{-1}(\omega)=-\frac{\gamma_{s} \omega_{p}^{2} / \omega^{3}+\epsilon_{d}^{\prime \prime}(\omega)}{\left[\epsilon^{\prime}(\omega)+2 \epsilon_{m}\right]^{2}+\left[\gamma_{s} \omega_{p}^{2} / \omega^{3}+\epsilon_{d}^{\prime \prime}(\omega)\right]^{2}} \simeq-\frac{\omega_{s}^{2}}{\epsilon_{d}^{\prime}\left(\omega_{s}\right)+2 \epsilon_{m}} \frac{\omega_{s} \gamma}{\left(\omega^{2}-\omega_{s}^{2}\right)^{2}+\omega_{s}^{2} \gamma^{2}},
$$

where $\omega_{s} \equiv \omega_{1}=\omega_{p} / \sqrt{\epsilon_{d}^{\prime}\left(\omega_{s}\right)+2 \epsilon_{m}}$ and $\gamma=\gamma_{s}+\omega_{s} \epsilon_{d}^{\prime \prime}\left(\omega_{s}\right)$ are the SP frequency and width, respectively. For typical widths $\gamma \ll \omega_{s}$, the integral in Eq. (28) can be easily evaluated, yielding

$$
\operatorname{Im} \Sigma_{\alpha}^{c}(\omega)=-\frac{24 e^{2} \omega_{s} E_{0}^{2}}{\epsilon_{d}^{\prime}\left(\omega_{s}\right)+2 \epsilon_{m}} \frac{E_{\alpha}^{c} \sqrt{2 m\left(\omega-\omega_{s}\right)}}{\left(\omega-E_{\alpha}^{c}-\omega_{s}\right)^{4}}\left[1-f\left(\omega-\omega_{s}\right)\right]
$$


Finally, using the relation $e^{2} k_{F}\left[\epsilon_{d}^{\prime}\left(\omega_{s}\right)+2 \epsilon_{m}\right]^{-1}=3 \pi \omega_{s}^{2} / 8 E_{F}$, the $\mathrm{SP}$-mediated scattering rate, $\gamma_{e}^{s}\left(E_{\alpha}^{c}\right)=-\operatorname{Im} \Sigma_{\alpha}^{c}\left(E_{\alpha}^{c}\right)$, takes the form

$$
\gamma_{e}^{s}(E)=9 \pi \frac{E_{0}^{2}}{\omega_{s}} \frac{E}{E_{F}}\left(\frac{E-\omega_{s}}{E_{F}}\right)^{1 / 2}\left[1-f\left(E-\omega_{s}\right)\right] .
$$

Recalling that $E_{0}=\left(2 m R^{2}\right)^{-1}$, we see that the scattering rate of a conduction electron is size-dependent: $\gamma_{e}^{s} \propto R^{-4}$. At $E=E_{F}+\omega_{s}$, the scattering rate jumps to the value $9 \pi\left(1+\omega_{s} / E_{F}\right) E_{0}^{2} / \omega_{s}$, and then increases with energy as $E^{3 / 2}$ (for $\omega_{s} \ll E_{F}$ ). This should be contrasted with the usual (bulk) plasmon-mediated scattering, originating from the first term in Eq. (22), with the rate decreasing as $E^{-1 / 2}$ above the onset.26 To estimate the size at which $\gamma_{e}^{s}$ becomes important, we should compare it with the Fermi liquid $e-e$ scattering rate, $23 \gamma_{e}(E)=\frac{\pi^{2} q_{T F}}{16 k_{F}} \frac{\left(E-E_{F}\right)^{2}}{E_{F}}$. For energies $E \sim E_{F}+\omega_{s}$, the two rates become comparable for

$$
\left(k_{F} R\right)^{2} \simeq 12 \frac{E_{F}}{\omega_{s}}\left(1+\frac{E_{F}}{\omega_{s}}\right)^{1 / 2}\left(\frac{k_{F}}{\pi q_{T F}}\right)^{1 / 2} .
$$

In the case of a $\mathrm{Cu}$ nanoparticle with $\omega_{s} \simeq 2.2 \mathrm{eV}$, we obtain $k_{F} R \simeq 8$, which corresponds to the radius $R \simeq 3 \mathrm{~nm}$. At the same time, in this energy range, the width $\gamma_{e}^{s}$ exceeds the mean level spacing $\delta$, so that the energy spectrum is still continuous. The strong size dependence of $\gamma_{e}^{s}$ indicates that, although $\gamma_{e}^{s}$ increases with energy slower than $\gamma_{e}$, the SP-mediated scattering should dominate for nanometer-sized particles. Note that the size and energy dependences of scattering in different channels are similar. Therefore, the total scattering rate as a function of energy will represent a series of steps at the collective excitation energies $E=\omega_{L}<\omega_{p}$ on top of a smooth energy increase. We expect that this effect could be observed experimentally in time--resolved two-photon photoemission measurements of sizeselected cluster beams.28

We now turn to the interband processes in noble metal particles and consider the scattering of a $d$-hole into the conduction band. From now on we restrict ourselves to the scattering via the dipole channel, mediated by the SP. The corresponding surface-induced potential, given by the $L=1$ term in Eq. (22), has the form 


$$
U_{s}\left(\omega ; \mathbf{r}, \mathbf{r}^{\prime}\right)=\frac{3 e^{2}}{R} \frac{\mathbf{r} \cdot \mathbf{r}^{\prime}}{R^{2}} \frac{1}{\epsilon_{s}(\omega)}
$$

With this potential, the $d$-hole self-energy is given by

$$
\Sigma_{\alpha}^{d}(i \omega)=-\frac{3 e^{2}}{R^{3}} \sum_{\alpha^{\prime}}\left|\mathbf{d}_{\alpha \alpha^{\prime}}\right|^{2} \frac{1}{\beta} \sum_{i \omega^{\prime}} \frac{G_{\alpha^{\prime}}^{c}\left(i \omega^{\prime}+i \omega\right)}{\epsilon_{s}\left(i \omega^{\prime}\right)}
$$

where $\mathbf{d}_{\alpha \alpha^{\prime}}=\left\langle c, \alpha|\mathbf{r}| d, \alpha^{\prime}\right\rangle=\left\langle c, \alpha|\mathbf{p}| d, \alpha^{\prime}\right\rangle / \operatorname{im}\left(E_{\alpha}^{c}-E_{\alpha^{\prime}}^{d}\right)$ is the interband transition matrix element. Since the final state energies in the conduction band are high (in the case of interest here, they are close to the Fermi level), the matrix element can be approximated by the bulk-like expression $\left\langle c, \alpha|\mathbf{p}| d, \alpha^{\prime}\right\rangle=\delta_{\alpha \alpha^{\prime}}\langle c|\mathbf{p}| d\rangle \equiv \delta_{\alpha \alpha^{\prime}} \mu$, the corrections due to surface scattering being suppressed by a factor of $\left(k_{F} R\right)^{-1} \ll 1$. After performing the frequency summation, we obtain for $\operatorname{Im} \Sigma_{\alpha}^{d}$

$$
\operatorname{Im} \Sigma_{\alpha}^{d}(\omega)=-\frac{9 e^{2} \mu^{2}}{m^{2}\left(E_{\alpha}^{c d}\right)^{2} R^{3}} \operatorname{Im} \frac{N\left(E_{\alpha}^{c}-\omega\right)+f\left(E_{\alpha}^{c}\right)}{\epsilon_{s}\left(E_{\alpha}^{c}-\omega\right)},
$$

with $E_{\alpha}^{c d}=E_{\alpha}^{c}-E_{\alpha}^{d}$. We see that the scattering rate of a $d$-hole with energy $E_{\alpha}^{d}, \gamma_{h}^{s}\left(E_{\alpha}^{d}\right)=$ $\operatorname{Im} \Sigma_{\alpha}^{d}\left(E_{\alpha}^{d}\right)$, has a strong $R^{-3}$ dependence on the nanoparticle size, which is, however, different from that of the intraband scattering, Eq. (32).

The important difference between the interband and the intraband $\mathrm{SP}$-mediated scattering rates lies in their energy dependence. Since the surface-induced potential, Eq. (34), allows for only vertical (dipole) interband single-particle excitations, the phase space for the scattering of a $d$-hole with energy $E_{\alpha}^{d}$ is restricted to a single final state in the conduction band with energy $E_{\alpha}^{c}$. As a result, the $d$-hole scattering rate, $\gamma_{h}^{s}\left(E_{\alpha}^{d}\right)$, exhibits a peak as the difference between the energies of final and initial states, $E_{\alpha}^{c d}=E_{\alpha}^{c}-E_{\alpha}^{d}$, approaches the SP frequency $\omega_{s}$ [see Eq. (36)]. In contrast, the energy dependence of $\gamma_{e}^{s}$ is smooth due the larger phase space available for scattering in the conduction band. This leads to the additional integral over final state energies in Eq. (28), which smears out the SP resonant enhancement of the intraband scattering.

As we show in the next section, the fact that the scattering rate of a $d$-hole is dominated by the SP resonance, affects strongly the nonlinear optical dynamics in small nanoparticles. 
This is the case, in particular, when the SP frequency, $\omega_{s}$, is close to the onset of interband transitions, $\Delta$, as, e.g., in $\mathrm{Cu}$ and $\mathrm{Au}$ nanoparticles 1.11.13.1.5 Consider an $e-h$ pair with excitation energy $\omega$ close to $\Delta$. As we discussed, the $d$-hole can scatter into the conduction band by emitting a SP. According to Eq. (36), for $\omega \sim \omega_{s}$, this process will be resonantly enhanced. At the same time, the electron can scatter in the conduction band via the usual two-quasiparticle process. For $\omega \sim \Delta$, the electron energy is close to $E_{F}$, and its scattering rate is estimated as $28 \gamma_{e} \sim 10^{-2} \mathrm{eV}$. Using the bulk value of $\mu, 2 \mu^{2} / m \sim 1 \mathrm{eV}$ near the L-point,29 we find that $\gamma_{h}^{s}$ exceeds $\gamma_{e}$ for $R \lesssim 2.5 \mathrm{~nm}$. In fact, one would expect that, in nanoparticles, $\mu$ is larger than in the bulk due to the localization of the conduction electron wave-functions. 1

\section{SURFACE PLASMON NONLINEAR OPTICAL DYNAMICS}

In this section, we study the effect of the SP-mediated interband scattering on the nonlinear optical dynamics in noble metal nanoparticles. When the hot electron distribution has already thermalized and the electron gas is cooling to the lattice, the transient response of a nanoparticle can be described by the time-dependent absorption coefficient $\alpha(\omega, t)$,

given by Eq. (2) with time-dependent temperature.30 In noble-metal particles, the temperature dependence of $\alpha$ originates from two different sources. First is the phonon-induced correction to $\gamma_{s}$, which is proportional to the lattice temperature $T_{l}(t)$. As mentioned in the Introduction, for small nanoparticles this effect is relatively weak. Second, near the onset of the interband transitions, $\Delta$, the absorption coefficient depends on the electron temperature $T(t)$ via the interband dielectric function $\epsilon_{d}(\omega)$ [see Eqs. (2) and (3)]. In fact, in $\mathrm{Cu}$ or $\mathrm{Au}$ nanoparticles, $\omega_{s}$ can be tuned close to $\Delta$, so the SP damping by interband $e-h$ excitations leads to an additional broadening of the absorption peak 1 In this case, the temperature dependence of $\epsilon_{d}(\omega)$ dominates the pump-probe dynamics. Below we show that, near the SP resonance, both the temperature and frequency dependence of $\epsilon_{d}(\omega)=1+4 \pi \chi_{d}(\omega)$ are strongly affected by the SP-mediated interband scattering. 
For non-interacting electrons, the interband susceptibility, $\chi_{d}(i \omega)=\tilde{\chi}_{d}(i \omega)+\tilde{\chi}_{d}(-i \omega)$, has the standard forme

$$
\tilde{\chi}_{d}(i \omega)=-\sum_{\alpha} \frac{e^{2} \mu^{2}}{m^{2}\left(E_{\alpha}^{c d}\right)^{2}} \frac{1}{\beta} \sum_{i \omega^{\prime}} G_{\alpha}^{d}\left(i \omega^{\prime}\right) G_{\alpha}^{c}\left(i \omega^{\prime}+i \omega\right),
$$

where $G_{\alpha}^{d}\left(i \omega^{\prime}\right)$ is the Green function of a $d$-electron. Since the $d$-band is fully occupied, the only allowed SP-mediated interband scattering is that of the $d$-hole. We assume here, for simplicity, a dispersionless $d$-band with energy $E^{d}$. Substituting $G_{\alpha}^{d}\left(i \omega^{\prime}\right)=\left[i \omega^{\prime}-E^{d}+E_{F}-\right.$ $\left.\Sigma_{\alpha}^{d}\left(i \omega^{\prime}\right)\right]^{-1}$, with $\Sigma_{\alpha}^{d}(i \omega)$ given by Eq. (35), and performing the frequency summation, we obtain

$$
\tilde{\chi}_{d}(\omega)=\frac{e^{2} \mu^{2}}{m^{2}} \int \frac{d E^{c} g\left(E^{c}\right)}{\left(E^{c d}\right)^{2}} \frac{f\left(E^{c}\right)-1}{\omega-E^{c d}+i \gamma_{h}^{s}\left(\omega, E^{c}\right)},
$$

where $g\left(E^{c}\right)$ is the density of states of conduction electrons. Here $\gamma_{h}^{s}\left(\omega, E^{c}\right)=\operatorname{Im} \Sigma^{d}\left(E^{c}-\omega\right)$ is the scattering rate of a $d$-hole with energy $E^{c}-\omega$, for which we obtain from Eq. (36),

$$
\gamma_{h}^{s}\left(\omega, E^{c}\right)=-\frac{9 e^{2} \mu^{2}}{m^{2}\left(E^{c d}\right)^{2} R^{3}} f\left(E^{c}\right) \operatorname{Im} \frac{1}{\epsilon_{s}(\omega)},
$$

where we neglected $N(\omega)$ for frequencies $\omega \sim \omega_{s} \gg k_{B} T$. Remarkably, $\gamma_{h}^{s}\left(\omega, E^{c}\right)$ exhibits a sharp peak as a function of the frequency of the probe optical field. The reason for this is that the scattering rate of a $d$-hole with energy $E$ depends explicitly on the difference between the final and initial states, $E^{c}-E$, as discussed in the previous section: therefore, for a $d$-hole with energy $E=E^{c}-\omega$, the dependence on the final state energy, $E^{c}$, cancels out in $\epsilon_{s}\left(E^{c}-E\right)$ [see Eq. (36)]. This implies that the optically-excited $d$-hole experiences a resonant scattering into the conduction band as the probe frequency $\omega$ approaches the SP frequency. It is important to note that $\gamma_{h}^{s}\left(\omega, E^{c}\right)$ is, in fact, proportional to the absorption coefficient $\alpha(\omega)$ [see Eq. (2)]. Therefore, the calculation of the absorption spectrum is a self-consistent problem defined by Eqs. (2), (3), (38), and (39).

It should be emphasized that the effect of $\gamma_{h}^{s}$ on $\epsilon_{d}^{\prime \prime}(\omega)$ increases with temperature. Indeed, the Fermi function in the rhs of Eq. (39) implies that $\gamma_{h}^{s}$ is small unless $E^{c}-E_{F} \lesssim k_{B} T$. Since the main contribution to $\tilde{\chi}_{d}^{\prime \prime}(\omega)$ comes from energies $E^{c}-E_{F} \sim \omega-\Delta$, the $d$-hole 
scattering becomes efficient for electron temperatures $k_{B} T \gtrsim \omega_{s}-\Delta$. As a result, near the SP resonance, the time evolution of the differential absorption, governed by the temperature dependence of $\alpha$, becomes strongly size-dependent, as we illustrate in the rest of this section.

In the numerical calculations below, we adopt the parameters of the experiment of Ref. 11, which was performed on $R \simeq 2.5 \mathrm{~nm} \mathrm{Cu}$ nanoparticles with $\mathrm{SP}$ frequency, $\omega_{s} \simeq 2.22 \mathrm{eV}$, slightly above the onset of the interband transitions, $\Delta \simeq 2.18 \mathrm{eV}$. In order to describe the time-evolution of the differential absorption spectra, we first need to determine the timedependence of the electron temperature, $T(t)$, due to the relaxation of the electron gas to the lattice. For this, we employ a simple two-temperature model, defined by heat equations for $T(t)$ and the lattice temperature $T_{l}(t)$ :

$$
\begin{gathered}
C(T) \frac{\partial T}{\partial t}=-G\left(T-T_{l}\right) \\
C_{l} \frac{\partial T_{l}}{\partial t}=G\left(T-T_{l}\right)
\end{gathered}
$$

where $C(T)=\Gamma T$ and $C_{l}$ are the electron and lattice heat capacities, respectively, and $G$ is the electron-phonon coupling. 31 The parameter values used here were $G=3.5 \times 10^{16}$ $\mathrm{Wm}^{-3} \mathrm{~K}^{-1}, \Gamma=70 \mathrm{Jm}^{-3} \mathrm{~K}^{-2}$, and $C_{l}=3.5 \mathrm{Jm}^{-3} \mathrm{~K}^{-1}$. The values of $\gamma_{s}$ and $\mu$ were extracted from the fit to the linear absorption spectrum, and the initial condition for Eq. (40) was taken as $T_{0}=800 \mathrm{~K}$, the estimated pump-induced hot electron temperature.11 We then selfconsistently calculated the time-dependent absorption coefficient $\alpha(\omega, t)$, and the differential transmission is proportional to $\alpha_{r}(\omega)-\alpha(\omega, t)$, where $\alpha_{r}(\omega)$ was calculated at the room temperature.

In Fig. 1 we plot the calculated differential transmission spectra for different nanoparticle sizes. Fig. 1(a) shows the spectra at several time delays for $R=5.0 \mathrm{~nm}$; in this case, the $\mathrm{SP}$-mediated $d$-hole scattering has no significant effect. Note that it is necessary to include the intraband $e-e$ scattering in order to reproduce the differential transmission lineshape observed in the experiment 11 For optically excited electron energy close to $E_{F}$, this can be achieved by adding the $e-e$ scattering rate $23 \gamma_{e}\left(E^{c}\right) \propto\left[1-f\left(E^{c}\right)\right]\left[\left(E^{c}-E_{F}\right)^{2}+\left(\pi k_{B} T\right)^{2}\right]$ to $\gamma_{h}^{s}$ in Eq. (38). The difference in $\gamma_{e}\left(E^{c}\right)$ for $E^{c}$ below and above $E_{F}$ leads to a lineshape 
similar to that expected from the combination of red-shift and broadening.

In Figs. 1(b) and (c) we show the differential transmission spectra with decreasing nanoparticle size. For $R=2.5 \mathrm{~nm}$, the apparent red-shift is reduced [see Fig. 2(b)]. This change can be explained as follows. Since here $\omega_{s} \sim \Delta$, the SP is damped by the interband excitations for $\omega>\omega_{s}$, so that the absorption peak is asymmetric. The $d$-hole scattering with the SP enhances the damping; however, since the $\omega$-dependence of $\gamma_{h}^{s}$ follows that of $\alpha$, this effect is larger above the resonance. On the other hand, the efficiency of scattering increases with temperature, as discussed above. Therefore, for short time delays, the increase in the absorption is relatively larger for $\omega>\omega_{s}$. With decreasing size, the strength of this effect increases, leading to an apparent blue-shift [see Fig. 2(c)]. Such a strong change in the absorption dynamics originates from the $R^{-3}$ dependence of the $d$-hole scattering rate; reducing the size by the factor of two results in an enhancement of $\gamma_{h}^{s}$ by an order of magnitude.

In Fig. 2 we show the time evolution of the differential transmission at several frequencies close to $\omega_{s}$. It can be seen that the relaxation is slowest at the SP resonance; this characterizes the robustness of the collective mode, which determines the peak position, versus the single-particle excitations, which determine the resonance width. For larger sizes, at which $\gamma_{h}^{s}$ is small, the change in the differential transmission decay rate with frequency is smoother above the resonance [see Fig. 2(a)]. This stems from the asymmetric lineshape of the absorption peak, mentioned above: the absorption is larger for $\omega>\omega_{s}$, so that its relative change with temperature is weaker. For smaller nanoparticle size, the decay rates become similar above and below $\omega_{s}$ [see Fig. 2(b)]. This change in the frequency dependence is related to the stronger SP damping for $\omega>\omega_{s}$ due to the $d$-hole scattering, as discussed above. Since this additional damping is reduced with decreasing temperature, the relaxation is faster above the resonance, compensating the relatively weaker change in the absorption. This rather "nonlinear" relation between the time-evolution of the pump-probe signal and that of the temperature, becomes even stronger for smaller sizes [see Fig. 2(c)]. In this case, the frequency dependence of the differential transmission decay below and above $\omega_{s}$ is 
reversed. Note, that a frequency dependence consistent with our calculations presented in Fig. 2(b) was, in fact, observed in the experiment of Ref. 11. At the same time, the changes in the linear absorption spectrum are relatively small.

\section{CONCLUSIONS}

To summarize, we have examined theoretically the role of size-dependent correlations in the electron relaxation in small metal particles. We identified a new mechanism of quasiparticle scattering, mediated by collective surface excitations, which originates from the surface-induced dynamical screening of the $e-e$ interactions. The behavior of the corresponding scattering rates with varying energy and temperature differs substantially from that in the bulk metal. In particular, in noble metal particles, the energy dependence of the $d$-hole scattering rate was found similar to that of the absorption coefficient. This led us to a self-consistent scheme for the calculation of the absorption spectrum near the surface plasmon resonance.

An important aspect of the SP-mediated scattering is its strong dependence on size. Our estimates show that it becomes comparable to the usual Fermi-liquid scattering in nanometer-sized particles. This size regime is, in fact, intermediate between "classical" particles with sizes larger than $10 \mathrm{~nm}$, where the bulk-like behavior dominates, and very small clusters with only dozens of atoms, where the metallic properties are completely lost. Although the static properties of nanometer-sized particles are also size-dependent, the deviations from their bulk values do not change the qualitative features of the electron dynamics. In contrast, the size-dependent many-body effects, studied here, do affect the dynamics in a significant way during time scales comparable to the relaxation times. As we have shown, the $\mathrm{SP}$-mediated interband scattering reveals itself in the transient pumpprobe spectra. In particular, as the nanoparticle size decreases, the calculated time-resolved differential absorption develops a characteristic lineshape corresponding to a resonance blueshift. At the same time, near the SP resonance, the scattering leads to a significant change 
in the frequency dependence of the relaxation time of the pump-probe signal, consistent with recent experiments. These results indicate the need for a systematic experimental studies of the size-dependence of the transient nonlinear optical response, as we approach the transition from boundary-constrained nanoparticles to molecular clusters.

The authors thank J.-Y. Bigot for valuable discussions. This work was supported by NSF CAREER award ECS-9703453, and, in part, by ONR Grant N00014-96-1-1042 and by Hitachi Ltd. 


\section{REFERENCES}

${ }^{1}$ See, e.g., U. Kreibig and M. Vollmer, Optical Properties of Metal Clusters (Springer, Berlin, 1995), and references therein.

${ }^{2}$ See, e.g., W. A. De Heer, Rev. Mod. Phys. 65, 611 (1993).

${ }^{3}$ See, e.g., M. Brack, Rev. Mod. Phys. 65, 677 (1993).

${ }^{4}$ See, e.g., Physics and Chemistry of Finite Systems: From clusters to Crystals, edited by P. Jena et al. (NATO Advanced Study Institute Series C, Kluwer Academic, Dordrecht/Boston, 1992).

${ }^{5}$ A. Kawabata and R. Kubo, J. Phys. Soc. Japan 211765 (1966).

${ }^{6}$ F. Hache, D. Ricard, and C. Flytzanis, J. Opt. Soc. Am. B 31647 (1986).

${ }^{7}$ G. S. Agarwal and S. D. Gupta, Phys. Rev. A 385678 (1988).

${ }^{8}$ L. Yang, K. Becker, F. M. Smith, R. H. Magruder, R. F. Haglund, L. Yang, R. Dorsinville, R. R. Alfano, and R. A. Zuhr, J. Opt. Soc. Am. B 11457 (1994).

${ }^{9}$ T. Tokizaki, A. Nkamura, S. Kaneko, K. Uchida, S. Omi, H. Tanji, and Y. Asahara, Appl. Phys. Lett. 65, 941 (1994).

${ }^{10}$ T. W. Roberti, B. A. Smith, and J. Z. Zhang, J. Chem. Phys. 102, 3860 (1995).

${ }^{11}$ J.-Y. Bigot, J.-C. Merle, O. Cregut, and A. Daunois, Phys. Rev. Lett., 75, 4702 (1995).

12 T. S. Ahmadi, S. L. Logunov, and M. A. Elsayed, J. Phys. Chem. 100, 8053 (1996).

${ }^{13}$ M. Perner, P. Bost, G. von Plessen, J. Feldmann, U. Becker, M. Mennig, M. Schmitt, and H. Schmidt Phys. Rev. Lett. 78, 2192 (1997).

${ }^{14}$ M. Nisoli, S. Stragira, S. De Silvestri, A. Stella, P. Tognini, P. Cheyssac, and R. Kofman, Phys. Rev. Lett. 78, 3575 (1997). 
15 T. Klar, M. Perner, S. Grosse, G. von Plessen, W. Spirkl, and J. Feldmann, Phys. Rev. Lett. 80, 4249 (1998).

${ }^{16}$ T. V. Shahbazyan, I. E. Perakis, and J.-Y. Bigot, Phys. Rev. Lett. 81, 3120 (1998).

${ }^{17}$ W. S. Fann, R. Storz, and H. W. K. Tom, Phys. Rev. B 46, 13592 (1992).

${ }^{18}$ C. K. Sun, F. Vallée, L. H. Acioli, E. P. Ippen, and J. G. Fujimoto, Phys. Rev. B 50, 15 337 (1994).

${ }^{19}$ R. H. M. Groeneveld, R. Sprik, and A. Lagendijk, Phys. Rev. B 51, 11433 (1994).

${ }^{20}$ N. Del Fatti, R. Bouffanais, F. Vallée, and C. Flytzanis, Phys. Rev. Lett. 81, 922 (1998).

21 The effects of the spill out of the electron wave-functions beyond the nanoparticle classical boundary were discussed, e.g., in V. V. Krezin, Phys. Rep. 220, 1 (1992).

${ }^{22}$ U. Sivan, Y. Imry, and A. G. Aronov, Europhys. Lett. 28, 115 (1994).

${ }^{23}$ D. Pines and P. Nozieres, The theory of quantum liquids, (W. A. Benjamin, Inc., New York, 1966), Vol. I.

${ }^{24}$ In semiconductor quantum dots, where the discrete energy levels are well resolved, the quasiparticle scattering rate is similar to that in the bulk only for energies larger than some critical energy; see, e.g., B. I. Altshuler, Y. Gefen, A. Kamenev, and L. S. Levitov, Phys. Rev. Lett. 78, 2803 (1997).

${ }^{25}$ A. A. Lushnikov and A. J. Simonov, Z. Phys. 270, 17 (1974).

${ }^{26}$ See, e.g., G. D. Mahan, Many-Particle Physics (Plenum, New York, 1990).

${ }^{27}$ In very small particles, the difference in the positions of the effective boundaries for the conduction and $d$-band densities leads to a shift in the linear absorption peak; see A. Liebsch, Phys. Rev. B 48, 11317 (1993); V. V. Krezin, Phys. Rev. B 51, 1844 (1995).

${ }^{28}$ S. Ogawa, H. Nagano, and H. Petek, Phys. Rev. B 55, 10869 (1997). 
${ }^{29}$ H. Ehrenreich and H. R. Philipp, Phys. Rev. 128, 1622 (1962).

30 The notion of a time-dependent absorption coefficient can also be extended to the coherent regime, see I. E. Perakis, Chem. Phys. 210, 259 (1996); I. E. Perakis, I. Brener, W. H. Knox, and D. S. Chemla, J. Opt. Soc. Am. B 13, 1313 (1996).

${ }^{31}$ G. L. Easley, Phys. Rev. B 33, 2144 (1986). 


\section{FIGURES}

FIG. 1. Calculated differential transmission spectra at positive time delays for nanoparticles with (a) $R=5 \mathrm{~nm}$, (b) $R=2.5 \mathrm{~nm}$, and (c) $R=1.2 \mathrm{~nm}$.

FIG. 2. Temporal evolution of the differential transmission at frequencies close the SP reso-

nance for nanoparticles with (a) $R=5 \mathrm{~nm}$, (b) $R=2.5 \mathrm{~nm}$, and (c) $R=1.2 \mathrm{~nm}$. 


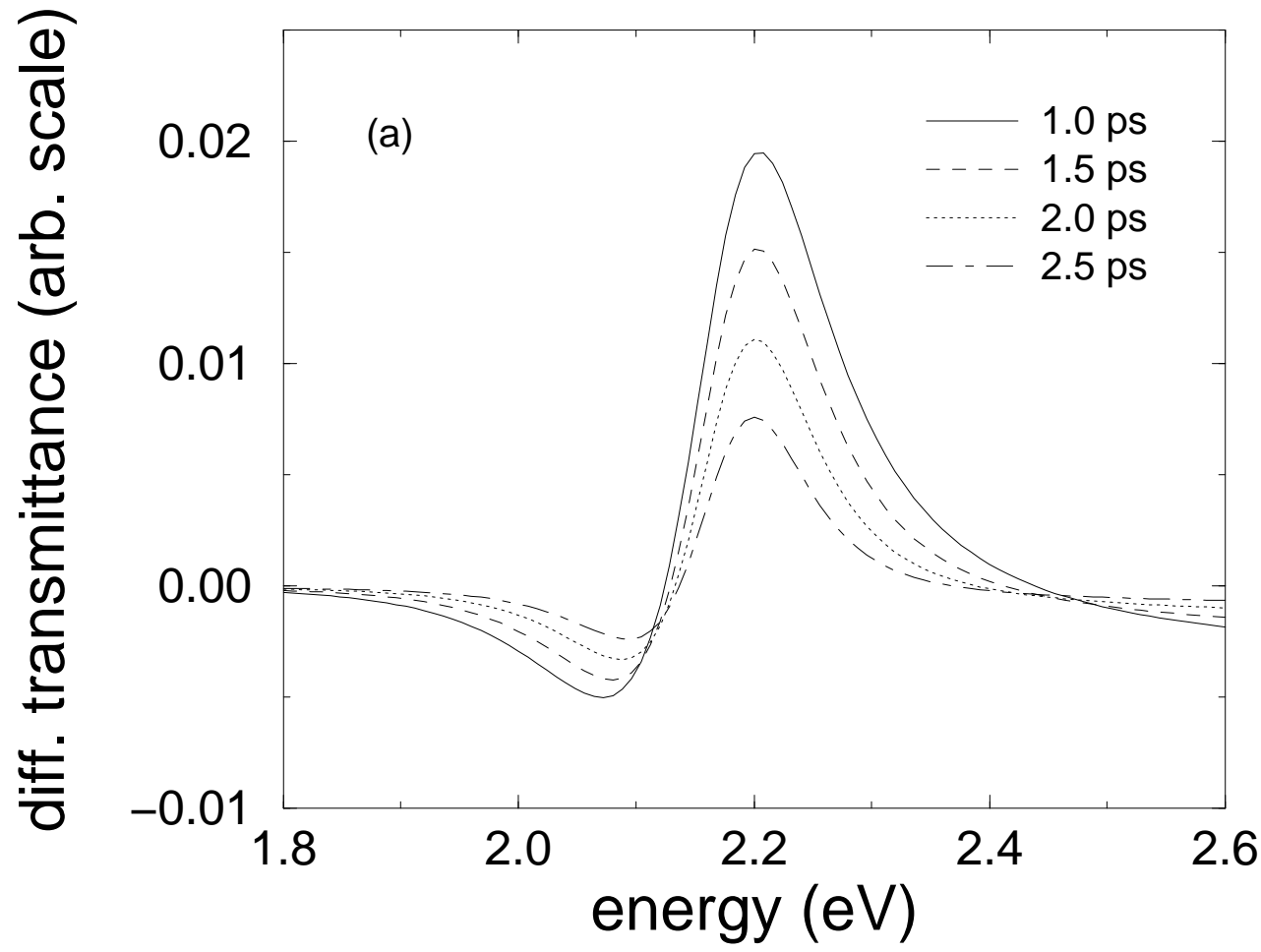

FIG. 1 


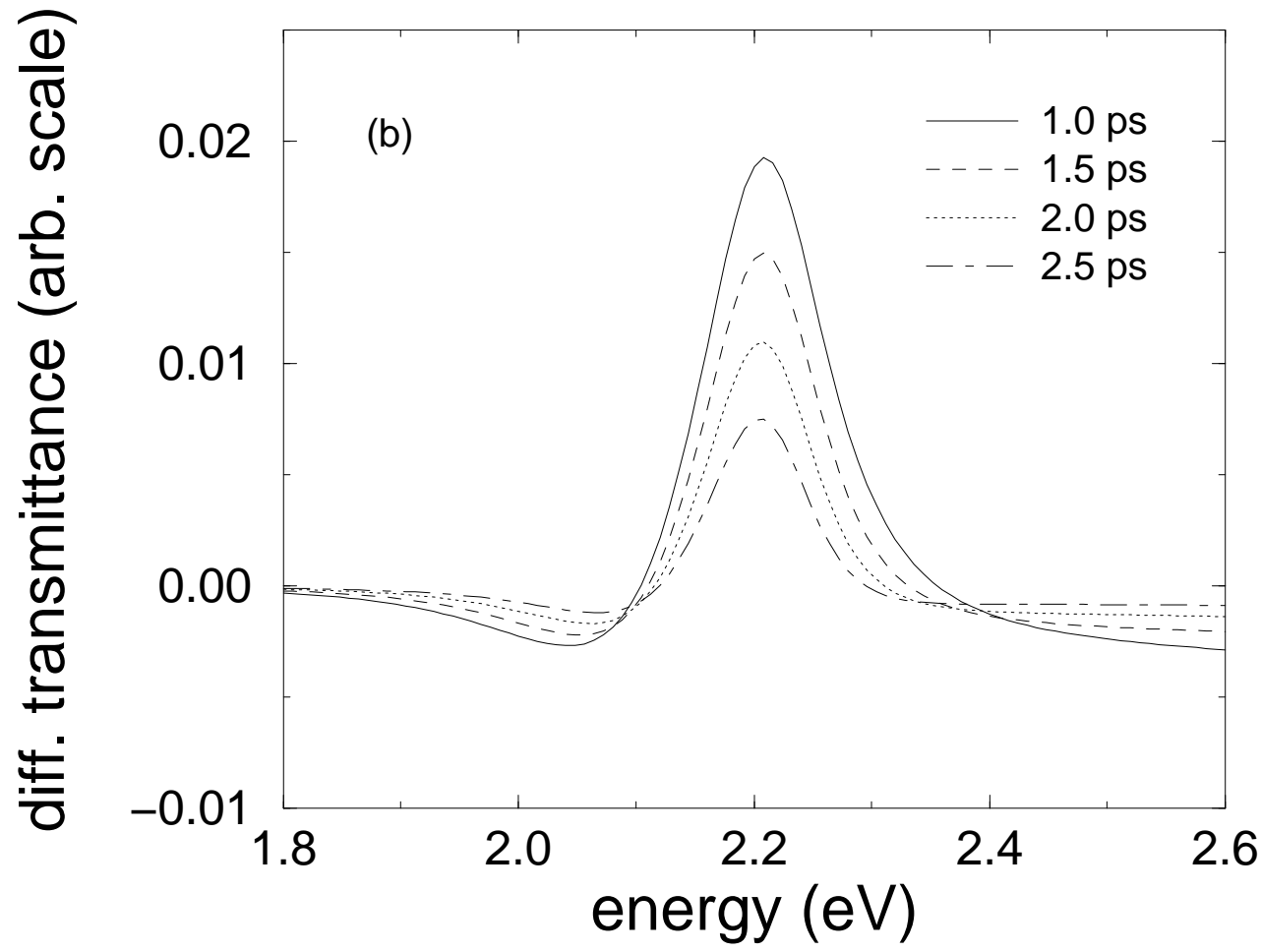

FIG. 1 


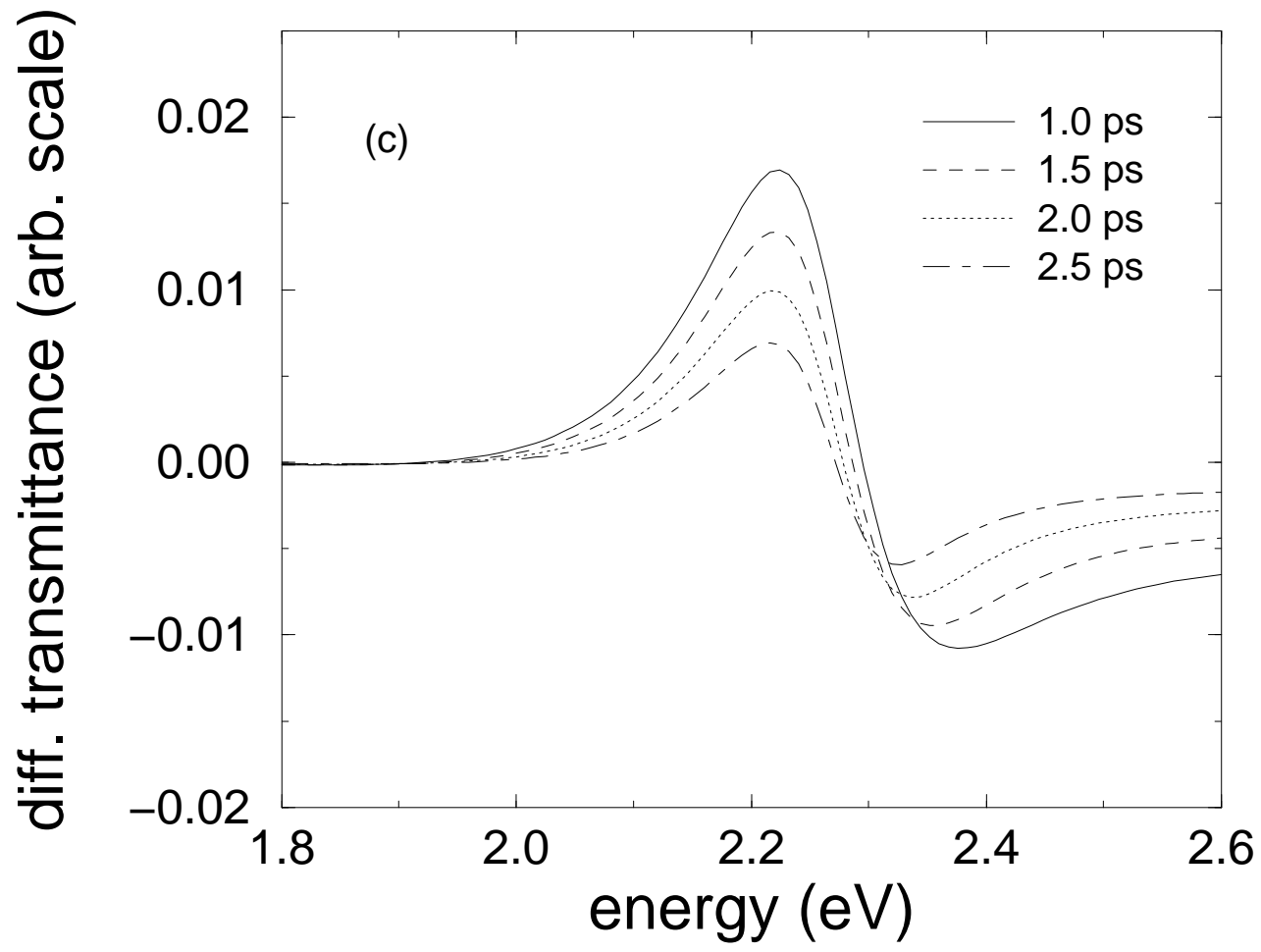

FIG. 1 


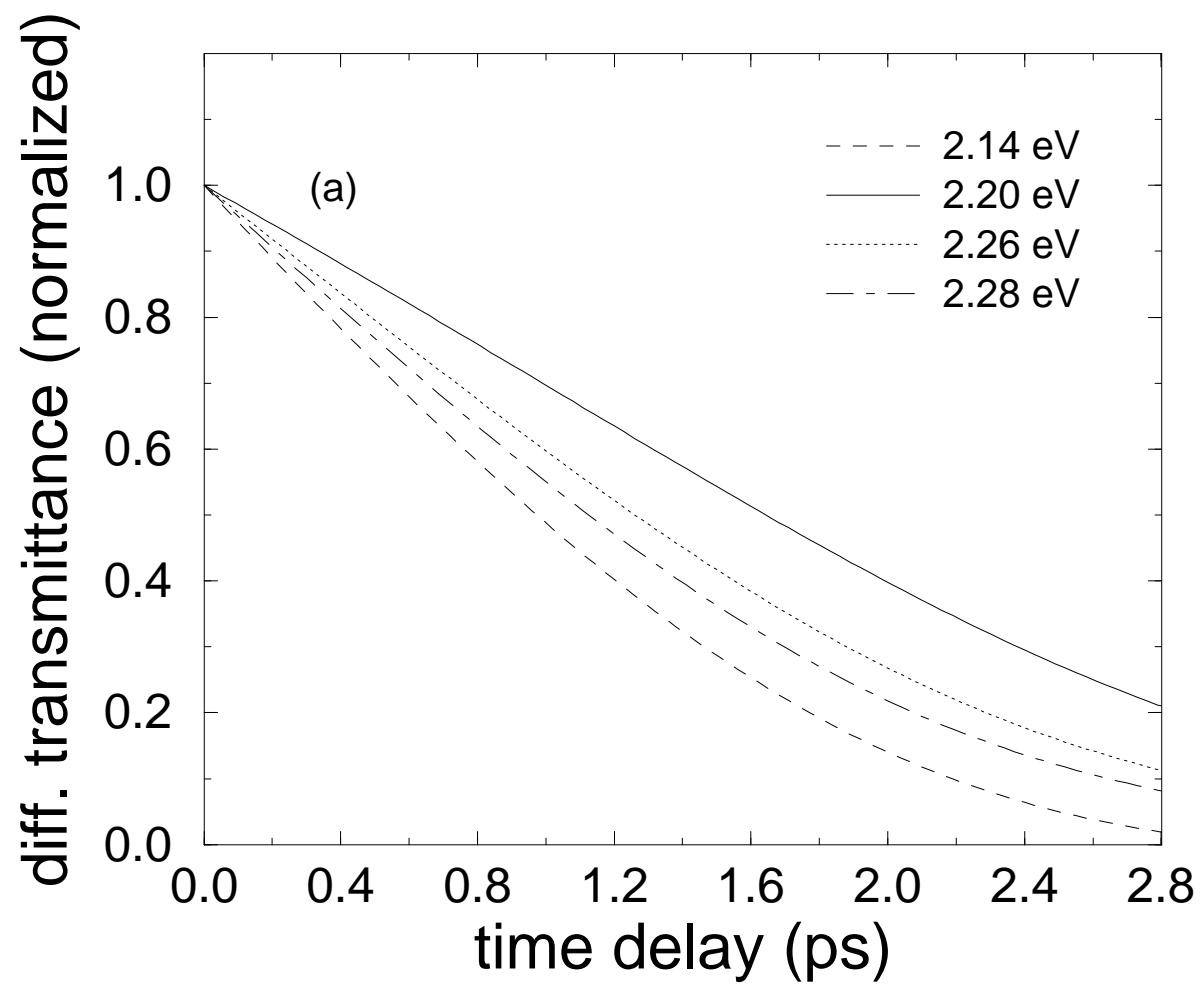

FIG. 2 


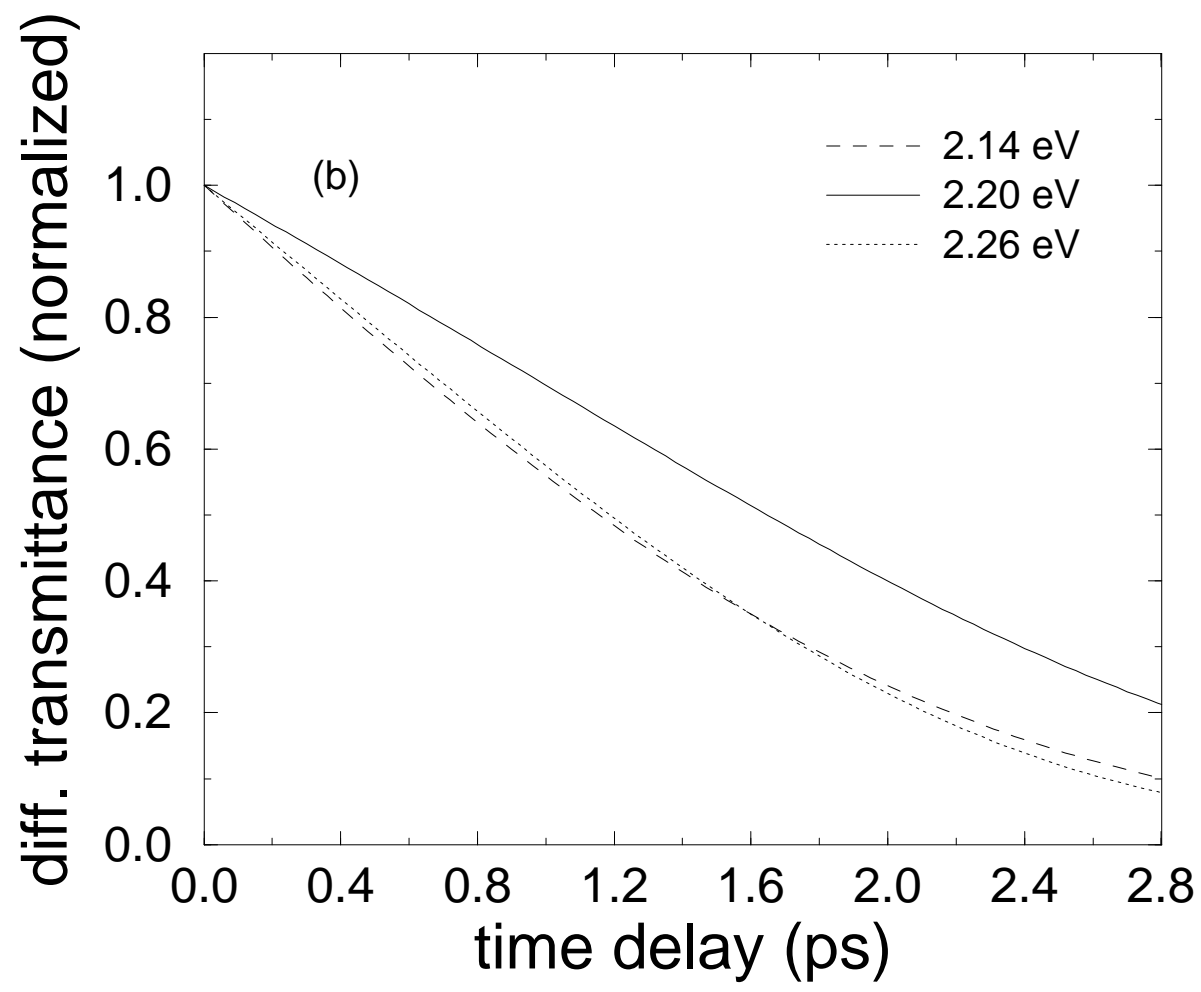

FIG. 2 


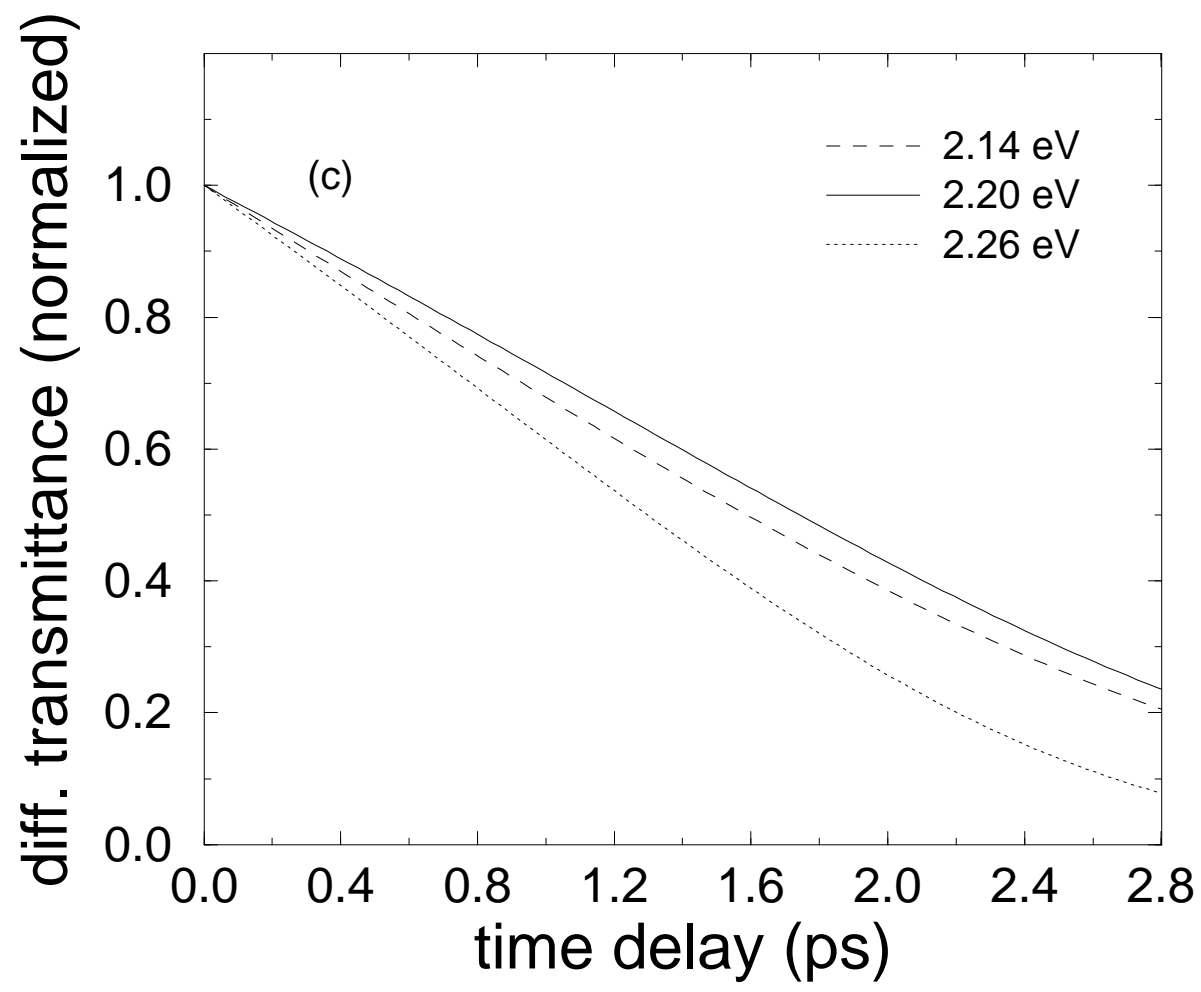

FIG. 2 